\title{
El cineforo: Estrategia didáctica en la generación de ingresos propios*
}

\author{
Campo-Redondo, María** \\ Árraga Barrios, Marisela***
}

\section{Resumen}

El presente artículo describe una experiencia de investigación-acción, en la cual se utilizó la modalidad de ciclos de cineforos como estrategia didáctica para generar ingresos propios en el Departamento de Psicología de la Escuela de Educación de la Facultad de Humanidades y Educación de la Universidad del Zulia (LUZ). Utilizando el concepto de cinemaeducación y a través de la implementación de ciclos de cineforos, se persiguieron dos objetivos fundamentales. Por un lado, se estudió la incidencia del cineforo en el desarrollo de un diseño de gestión administrativa que produjera nuevas formas de generar ingresos propios y por el otro, se concibió al cineforo como estrategia didáctica, que beneficiase en lo particular a los estudiantes del referido Departamento y a otros miembros de la comunidad universitaria. Se recoge que los resultados de esta experiencia de investigación indican que la modalidad de cineforo es productiva como actividad generadora de ingresos propios y como estrategia didáctica innovadora, empleando estímulos visuales, auditivos y experienciales. Se concluye que el cineforo resultó ser una estrategia didáctica extra-muros que promueve la participación activa, responsable y comprometida de las personas y entes involucrados en el proceso educativo, de forma innovadora, creativa e integradora.

Palabras clave: Generación de ingresos propios, cineforo, estrategia didáctica, cinemaeducación.

Recibido: 04-06-22. Aceptado: 05-02-01

* Parte de esta investigación se realizó con fondos provenientes del proyecto de investigación No. 750-04, del Consejo de Desarrollo Científico y Humanístico (CONDES) de la Universidad del Zulia.

** Psicólogo Clínico. Doctora en Ciencias Humanas. Profesora Titular del Departamento de Psicología y de la Maestría en Orientación. Investigadora adscrita al Centro de Documentación en Investigación Pedagógica (CEDIP). Universidad del Zulia.E-mail: marisucampo@yahoo.com

*** Orientadora. Magíster en Orientación. Candidata a Doctora en Ciencias Humanas. Profesora Asociada del Departamento de Psicología y de la Maestría en Orientación. Investigadora adscrita al Centro de Documentación en Investigación Pedagógica (CEDIP). Universidad del Zulia. E-mail: mariarraga@hotmail.com 


\title{
The Cinema-Forum: A Didactic Strategry for the Generation of Income
}

\begin{abstract}
This article describes a research-action experience, in which the modality of cinema-forums was used to generate income for the Psychology Department at the School of Education ant the University of Zulia (LUZ). Two fundamental objectives were sought, utilizing the concept of cinemaeducation through the implementation of cycles of cinema forums. On the one hand the incidence of cinema forums in the development of a design for administrative management in order to produce income for the department and for other members of the academic community was studied. The results of this research experience indicated that cinema-forums are productive as a sources of income, and as an innovative didactic strategy, employing visual, auditive and experiencial stimulus. The conclusion is that cinema-forums are external didactic strategies that promote active participation, responsibility, and compromise on the part of the people and the institutions involved in the educational process in an innovative, creative and integrated manner.
\end{abstract}

Key words: Income generation, cinema-forum, didactic strategy, cinema or film education.

\section{Introducción}

Los cambios en la sociedad global y en el sistema educativo han producido una serie de fenómenos de diferente naturaleza, desafiando a docentes e investigadores a comprender esa problemática y elaborar propuestas nuevas acordes a dichas circunstancias.

La crisis de las universidades venezolanas ha suscitado un cambio en la manera de abordar los procesos educativos y de generación de ingresos propios. Por un lado, en el campo educativo, cada vez con mayor frecuencia aparecen publicaciones (Flores, 1994; Nelson y Neufeldt, 1998; Vera, 2002) que describen experiencias donde se "deja" el aula, para favorecer el proceso enseñanza-aprendizaje en ambientes no tradicionales y con modalidades didácticas que se alejan de la clase magistral. Por el otro lado, en el ámbito gerencial, ha comenzada a germi- nar el concepto de la Nueva Gestión Pública (NGP), la cual busca la flexibilización de la organización, la descentralización (Ochoa, 2004) y la generación de ingresos propios (Velasco, 2002).

En este orden de ideas, ante la grave crisis de asignación presupuestaria que atraviesan las universidades del país, el Departamento de Psicología de la Escuela de Educación de la Facultad de Humanidades y Educación de la Universidad del Zulia se propuso llevar a cabo una experiencia de investigación-acción participativa que combinase estas dos dimensiones-problema: la creación de acciones que condujesen a la generación de ingresos propios y el desarrollo de actividades de carácter masivo y extramuros que sirvieran como estrategia didáctica complementaria a la clase magistral.

Para afrontar esta problemática, nos planteamos investigar qué estrategias podríamos emplear para: a) generar re- 
cursos bajo la modalidad de ingresos propios que pudieran ser invertidos en el Departamento de Psicología y b) promocionar y discutir temas relacionados con las asignaturas que administra nuestro Departamento, que favorecieran el aprendizaje en ambientes no tradicionales.

Esto nos llevó a la implementación del modelo de cinemaeducación (Alexander, 1995) a través de su estrategia didáctica el cineforo. Para ello, elaboramos un diseño específico que consistió en la planificación y puesta en marcha de ciclos de cineforos, a partir de una concepción constructivista, desde la cual se planteó la búsqueda de alternativas pedagógicas ajustadas a las particularidades de la estrategia didáctica extramuros y vivencial, como alternativa a la clase teórica magistral e intramuros.

Es importante resaltar que si bien existen publicaciones que reportan haber estudiado al cine como estrategia didáctica en el salón de clase, como por ejemplo las de Koch y Dollarhide (2000) y Higgins y Dermer (2001), hasta la fecha no encontramos experiencias de investigación que utilizasen ciclos completos de cineforos, administrados y ejecutados por un Departamento de Psicología, que fuesen llevados a cabo con el doble propósito de servir como insumo en la enseñaza de la orientación y la psicología; y ser al mismo tiempo una actividad generadora de ingresos propios.

De este planteamiento, surgió el objeto de estudio de nuestra investigación. Investigar al cineforo como:

- estrategia didáctica en la enseñanza de la orientación y la psicología

- actividad generadora de ingresos propios.

\section{El cineforo como estrategia didáctica en la enseñanza de la orientación}

Diversas publicaciones y artículos (por ejemplo Koch y Dollarhide, 2000) han aparecido en los últimos años donde vinculan al cine con la educación y muy especialmente con la orientación.

En este sentido, existe una tradición en educación de lo que se considera como cine didáctico. Incluso hay autores que han denominado a la estrategia de incluir cine en la educación de estudiantes de medicina y salud mental como "cinemaeducación", con el fin de educarlos en temas "sensibles", como la homosexualidad, multiculturalidad y salud mental entre otros (Alexander, 1995).

Higgins y Dermer (2001) plantean que la inserción del cine en el currículo de la educación de orientadores crea un ambiente de aprendizaje único y motivador para propiciar el desarrollo de competencias complejas relacionadas con la disciplina de la orientación y la psicología. El cine, reportan estas autoras, tiene una versatilidad y un "appeal" universal, al tiempo que sirve como actividad de entretenimiento económica y masiva.

Greenberg (1993), reporta el uso del cine en estudiantes residentes de psiquiatría, en programas de aprendizaje de psicoterapia y en institutos de formación psicoanalítica, para ilustrar los psicodinamismos y la psicopatología de los personajes del film. También Higgins y Dermer (2001) han tenido excelentes resultados en el uso del cine en la enseñanza de materias de orientación (counseling) tales como psicopatología y estudio de casos, 
y orientación familiar y de pareja, entre otras.

De igual modo, Alfredo (2004) comenta que el cine puede considerarse como uno de los factores más poderosos de creación de actitudes públicas y de difusión de ideas acerca de temas relacionados con la ciencia en general y con la promoción y orientación de la salud mental en particular.

Las imágenes de los científicos y de la actividad científica que pueblan el imaginario simbólico colectivo, han sido propagadas especialmente a través de los medios audiovisuales. En tal sentido, el cine ha contribuido a generar ciertas formas de pensar sobre los profesionales de servicio y orientación, roles sociales, valores y concepciones de la sexualidad $y$ del honor entre otros.

El cine puede representar una herramienta fecunda para estimular un interés crítico por temas de actualidad. Al respecto, Alfredo (2004) reporta que el principal reto de la utilización del cine como estrategia didáctica radica en ser capaces de posibilitar, a partir del impacto que las películas generan en el alumnado, un interés y preguntas pertinentes al tema de la película en cuestión. Así, el cine se puede convertir en una experiencia vivida de aprendizaje significativo. El cine contribuye a hacer más directo el conocimiento, en el sentido de que ayuda a incorporar conceptos aprendidos a nuestras propias experiencias vitales. Por ejemplo, conocer el concepto de la violencia familiar y poderlo escribir en un examen es una cosa. Otra bien distinta, es conocer "de cerca" los detalles de las relaciones que se producen en ambientes familiares donde la violencia es el modo de relación sobresaliente y en la cual se puede apreciar el impacto que este fenómeno tiene en la sociedad, o las resonancias con otras esferas de la vida cotidiana, como el trabajo y la salud física.

Al respecto, al analizar la película "Amores perros" (una de las proyectadas en el ciclo de cineforos), podemos no solo identificar estas características de la violencia familiar, sino "vivirlas" casi al mismo ritmo de los protagonistas de la obra.

En el caso concreto de la cinemaeducación, vemos que el cine tiene una capacidad de contribuir al conocimiento y concepción de la realidad social en función del grado de aproximación "realista" de las imágenes a los hechos. Se puede decir que el cine es una forma de producción de ideas que se relacionan con una cultura y un hecho social determinado.

La utilización del cine en la enseñanza de la orientación permite un acercamiento en torno al mundo de las imágenes. Enseñar a mirar una imagen, descodificarla, analizarla, hacer hermenéutica, es tan importante como saber leer y entender un texto escrito o atender a un caso para orientarlo. Así, estamos convencidas de que con la realización de cineforos en torno a temas relacionados con la enseñanza de la orientación (como por ejemplo, relaciones de pareja y dinámica familiar), podemos fomentar la capacidad crítica de los alumnos, al tiempo que estimulamos la creación de actitudes positivas hacia los temas de interés de nuestro departamento.

La necesidad de aplicar herramientas teóricas al análisis de los acontecimientos planteados en los films obliga, por un lado, a entrelazar y dar cohesión 
con lo adquirido, a acercar lo aprendido en los libros a contextos "prácticos" (la vivencia de la película), y por el otro, a revisar posibles identificaciones que hacemos con las tramas o personajes de la producción. En este sentido, el proceso de aprender y conocer, a través del cine, adquiere un carácter "real" y mucho más estimulante.

Desde un encuadre sociológico, la cinemaeducación reposa en una concepción de la educación como un proceso social y socializador, mediante el cual los grupos humanos (docentes-investigadores en este caso) tratan de promover el desarrollo de los más jóvenes (estudiantes de la mención Orientación). Este proceso, que siempre tiene una dinámica interna, tiene lugar en un medio social culturalmente organizado (los ciclos de cineforo y su posterior discusión en las clases intramuros) y es posible porque otras personas ejercen un papel mediador (los panelistas y la discusión que promueve el cineforo) entre la cultura (la participación en el cineforo y discusión de la película) y el individuo en el proceso de construcción del conocimiento, influyendo sobre dos tipos de adquisiciones: las capacidades generales y los aprendizajes específicos, ligados al medio cultural en el que se produce el conocimiento.

Ambas adquisiciones se encuentran profundamente relacionadas, lo que hace que el enfoque constructivista del cineforo proponga la organización de experiencias donde el alumno pueda construir significados culturales. El objetivo es el crecimiento cognoscitivo de los estudiantes mediante la construcción significativa de aprendizajes específicos relacionados con los temas de las películas discutidas.
La implementación de cineforos se ha ido desarrollando basándose en el auge creciente de los enfoques cognitivistas en el estudio del desarrollo humano, que subrayan el carácter constructivo del proceso de adquisición del conocimiento. El mismo implica la idea de un ser humano que selecciona, asimila, procesa, interpreta y confiere significados a los estímulos y configuraciones de estímulos.

En el caso de la enseñanza de la orientación, el texto fílmico se construye con la propia participación del espectadoralumno (la imagen mirada y sentida del cine), en la medida en la que éste produce y otorga sentido a las propuestas del realizador. Esto explica los procesos psicológicos de identificación y proyección. Así mismo, el cine posee una capacidad de representación de lo complejo que ayuda a sintetizar lo multidimensional del mundo en el que vivimos los seres humanos.

Por otra parte, la posibilidad de que panelistas especialistas en el área de la orientación o de la temática a abordada analicen cada film con puntos de vista diferentes, le ofrece al espectador-alumno la oportunidad de construir sus propias respuestas a los procesos socio-emocionales generados en ellos y a su propio mundo, lo que puede influir en el logro de aprendizajes significativos.

De lo antes discutido podemos inferir que lo que nos condujo a la experiencia de utilizar al cineforo como tecnología didáctica es el convencimiento de que lo importante no es la tecnología (en nuestro caso la del cine), sino utilizar la mejor tecnología didáctica disponible en cada momento al servicio de una idea: favorecer y universalizar el aprendizaje. Por lo tanto, era necesario no dejar ac- 
tuar por sí solo al cineforo por muy motivante y entretenida que fuese una película, sino poder influir a nuestros estudiantes a través de los films y su posterior discusión a lograr el resultado previsto en las intenciones educativas de nuestro Departamento.

Sintetizando lo antes expuesto, podemos afirmar que nuestro interés primordial en esta investigación fue el de utilizar una estrategia didáctica que favorezca el proceso educativo, produciendo un aprendizaje distinto al que se logra con tecnologías y en ambientes tradicionales.

\section{El cineforo como actividad generadora de ingresos propios}

Generar ingresos propios se ha convertido en una herramienta necesaria para enfrentar los desafíos académicos presentes en el sector universitario de nuestro país, dadas las limitaciones del presupuesto universitario y la necesidad de introducir estrategias innovadoras en el proceso educativo.

La importancia cuantitativa y cualitativa de la generación de ingresos propios en el sector universitario, hace imprescindible disponer de un programa que, partiendo de una reflexión sobre la realidad actual, constituya una clara apuesta por el futuro, que contribuya con el incremento en la calidad del proceso educativo, basado en la innovación, en los procesos creativos, en la investigación científica y tecnológica, y en la generación de recursos financieros como elemento de autonomía, creatividad, flexibilización de la organización y descentralización.
Al respecto, Ochoa (2004) expresa, que el concepto de la Nueva Gestión pública (NGP) busca la flexibilidad de la organización y la descentralización. Por su parte, Barzelay (2004) propone como elemento a considerar para quienes se acercan a trabajar en la noción de la Nueva Gestión Pública, en un mundo inmerso en procesos de intensa innovación tecnológica, redefinir las competencias centrales de la unidad de trabajo (en este caso sería el Departamento de Psicología) en torno a nuevas categorías estratégicas que le permitan adaptarse al futuro, implicando esto las decisiones sobre nuevas propuestas de trabajo y de generación de ingresos para la adquisición de equipamiento y gastos de investigación.

Esta redefinición de competencias de la que habla Barzelay, le proporciona flexibilidad y capacidad de transformación a la organización, al explotar las posibilidades de crear nuevas alternativas acordes con los cambios que se van suscitando, para generar mejoras en los procesos.

Velasco (2002) expone, que los ingresos propios representan un aspecto de la autogestión, que se concreta en la implantación de un modelo con la participación de los estudiantes y profesores de los departamentos de cada escuela, que permita la dotación de aulas y cátedras, que tiendan a facilitar el proceso enseñanza aprendizaje, de acuerdo a los requerimientos existentes.

El Departamento de Psicología, convencido de la necesidad de buscar nuevas estrategias didácticas que faciliten el desarrollo de competencias relacionadas con la orientación y la psicología, en un ambiente de participación que produz- 
ca ingresos para ser invertidos en nuevas actividades académicas que favorezcan el proceso enseñanza-aprendizaje, realizó un diseño de generación de ingresos propios por medio de las actividades del cine-foro, fundamentado en tres elementos: 1. Los contenidos inherentes a las asignaturas que administra el Departamento. 2. El concepto de participación y 3. Generación de ingresos propios.

1. Los contenidos inherentes a las asignaturas que administra el Departamento

En la búsqueda de creatividad y flexibilidad, el Departamento se propuso definir las asignaturas que pudieran trabajarse a través de una estrategia didáctica innovadora, como el cineforo, que generara ingresos para ser invertidos en equipos y dotación de material para los estudiantes. Se decidió trabajar con las asignaturas: "Psicología del Desarrollo", "Psicología Educativa", "Enfoque Sistémico de la Realidad", "Orientación Familiar", "Psicología Educativa" y "Orientación Personal", por considerar que todas estas unidades curriculares tienen entre sus contenidos temas de interés, que por su cotidianidad y profundidad pueden estudiarse a través del visionado y posterior análisis de películas; generando experiencias propias a través de los personajes de los films, que propicien procesos de identificación y búsqueda de alternativas a las situaciones experienciadas, mientras ayudan a generar ingresos que serán invertidos en equipos y actividades similares para el Departamento.

Se trata de un trabajo complejo que requiere el aporte de recursos humanos calificados para concebirlo y ponerlo en marcha, y el apoyo posterior de todos los organismos involucrados, tales como Consejo de Escuela y Facultad, organismos que avalaron y apoyaron la actividad, el equipo de profesores del Departamento de Psicología, quienes eligieron cuidadosamente los temas de estudio y el grupo de panelistas que dirigió la discusión, la Unidad de Extensión de la Facultad, quien ofreció el apoyo logístico: local, equipos de video y sonido, entre otros; y los estudiantes de la Escuela de Educación, el personal administrativo y el obrero.

2. El concepto de participación

El concepto de participación lo entendemos como el involucramiento psicológico y físico de las personas en situaciones de trabajo en equipo, que las estimula a contribuir a la obtención de las metas del equipo y a compartir la responsabilidad de éstas a partir de acuerdos que generan beneficios mutuos desde el punto de vista académico y económico (Velasco, 2002). En tal sentido, en los ciclos de cineforos realizados participaron además de los docentes y estudiantes de la mención de Orientación, las personas anteriormente mencionadas, tanto en el diseño de la actividad, como en su implementación y desarrollo. En lo que se refiere a la participación hay cuatro elementos importantes que se han considerado para generar ingresos: 1. El involucramiento, 2. La contribución, 3. La responsabilidad y 4 . Los beneficios mutuos. El involucramiento mental, emocional y físico en el sentido no sólo de la participación (de docentes del Departamento de Psicología y estudiantes de la mención Orientación, de la Escuela de Educación y demás personas del equipo) sino de un alto nivel de compromiso personal con las actividades programadas, referidas al cineforo. 
La contribución, se ha convertido en un medio que facilita la toma de iniciativa de los involucrados y los aportes creativos en la consecución de los objetivos que se persiguen: generar ingresos propios y emplear el cineforo como estrategia didáctica. En este aspecto, tanto los aportes de los profesores como de los estudiantes han sido imprescindibles en la consecución de los objetivos planteados para cada una de las actividades previstas en el cineforo. La responsabilidad, como tercer elemento importante, tiene que ver no sólo con el compromiso personal sino también grupal, con el trabajo en equipo, la coordinación de actividades y el engranaje de cada una de las tareas que se realizaron (Velasco, 2002).

Los beneficios mutuos es el último elemento; éste implica un trabajo arduo dirigido a la búsqueda del bien común en el sentido en que lo plantea Petrella (1997); representado por la existencia de los otros y de espacios de convivencia donde se puedan generar acuerdos y la garantía de beneficios para todos los miembros de la comunidad. En tal sentido, con la puesta en marcha de los ciclos de cineforos, se beneficiaron tanto los profesores y estudiantes que asistieron, como otras personas de la comunidad universitaria como extrauniversitaria.

3. Generación de ingresos propios

La generación de ingresos propios constituye el tercer elemento sobre el cual se fundamenta la actividad de cineforo y se refiere, según Velasco (2002) a la producción de ingresos económicos para ser invertidos en equipos, materiales y acondicionamiento de aulas y espacios destinados al desarrollo de actividades académicas que redunden en la calidad del pro- ceso enseñanza-aprendizaje y en la creación de nuevas estrategias educativas en el Departamento de Psicología.

La producción de ingresos propios debe considerar las necesidades del mercado y adecuarlas a las políticas y presupuesto de la empresa u organización, que en este caso es la Universidad del Zulia y el Departamento de Psicología, específicamente, de manera que se convierta en una actividad de servicio que permita responder a las necesidades reales de los involucrados en forma innovadora.

La razón práctica es que son los involucrados directamente en el proceso educativo, quienes conocen las necesidades que poseen y requieren atender. La generación de ingresos propios se convierte en una estrategia que permite cubrir las necesidades que surgen de manera imprevista y cuya satisfacción es puntual para mejorar la calidad del proceso enseñanza aprendizaje; así como aquellas otras que aunque han surgido en el tiempo y han sido previstas, requieren de asignaciones que el Estado venezolano no puede proveer por la deficiencia presupuestaria y el orden jerárquico en que ha previsto atender las situaciones educativas.

De estas consideraciones se puede inferir que la finalidad de la implementación del cineforo fue la de proponer una estrategia didáctica, generadora de ingresos propios para el Departamento de Psicología que permitiera la prestación de un servicio a la comunidad universitaria, específicamente a los estudiantes de la mención Orientación y en general a los de la Escuela de Educación para invertir recursos en el mejoramiento del proceso educativo, a través de la compra de equi- 
pos audiovisuales que faciliten el desarrollo de actividades académicas dentro y fuera del aula.

\section{Método}

Para lograr los objetivos planteados en esta investigación, se recurrió a la metodología de la investigación-acción, específicamente el de la investigaciónacción- en el-salón-de-clase (classroom-action-research en inglés) descrita por Kemmimis y Taggart (2000). El énfasis en este tipo de investigación se dirige a los aspectos "prácticos" que tanto el docente como los estudiantes hacen de la situación a estudiar.

El método de la investigación-acción en el-salón-de-clase generalmente involucra la utilización de modalidades cualitativas e interpretativas en el manejo de las experiencias de la investigación y sus resultados por parte del docente. Kemmimis y Taggart (2000), remarcan que la propuesta de la investigación-acción difiere de otras modalidades investigativas en que está mucho mas "interesada" en cambiar las prácticas particulares y específicas de los actores (docentes y modalidades de enseñanza en nuestro caso) que en cambiar las prácticas en abstracto o en general.

En la investigación-acción, el investigador se centra principalmente en acciones para mejorar la práctica docente. En este tipo de método, el investigador, quien a su vez es el docente, le da primacía al autoentendimiento de lo que él como docente pretende realizar para mejorar o potenciar el proceso de aprendizaje de sus alumnos. Así, los investigadores que se basan en la investigación- acción sólo pueden hacerla investigando las prácticas y "problemas" propios en "ellos mismos" y no en otros; de esto se deduce que el investigador que recurre a la investigación-acción no puede investigar distanciándose del objeto de estudio. En nuestro caso, las autoras de este trabajo fueron al mismo tiempo actores (docentes) y protagonistas (junto a sus estudiantes) del proceso investigativo.

Al respecto, Kemmimis y Taggart (2000) definen a la investigación-acción en el-salón-de-clase como una acción práctica donde el investigador-docente se pregunta cómo actuar "correctamente" y "adecuadamente" en la situación que el docente confronta a diario. Estos autores entienden la metodología basada en la investigación-acción como un proceso en el cual los actores (investigadores-docentes) se centran en entender cómo los actos de comunicación, producción y organización social (en nuestro caso el cineforo como estrategia didáctica) están entramadas en unas prácticas particulares (en este caso las de docencia en el Departamento de Psicología) con otros actores (en nuestro caso con los estudiantes) y en una acción colaborativa se producen cambios en la manera como se participa en el proceso (en nuestro caso el de enseñar de modo diferente y planificar actividades extramuros conducentes a la generación ingresos propios).

En este sentido, realizamos acciones que nos llevaran a entender nuestra práctica pedagógica en nuestro propio contexto (La Universidad del Zulia), con nuestros propios docentes y alumnos (Departamento de Psicología), con nuestras necesidades reales (prestar un servicio que produzca ingresos propios) y con 
nuestros propios recursos (planificación de actividades extracátedra), para generar cambios en nuestras estrategias didácticas (la cinemaeducación a través del cineforo) de modo tal que la comunidad se transformase (nuestros alumnos logren aprendizajes vivenciales y significativos sobre temas relacionados con la orientación y la psicología).

En nuestra investigación, como docentes-investigadoras nos interesamos en estudiar de manera exploratoria la puesta en práctica de ciclos de cineforos con el propósito de generar ingresos propios y al mismo tiempo ensayar una tecnología de enseñanza diferente y complementaria a la tradicional.

Como investigadoras-docentes influidas por la metodología de investigación-acción, recurrimos a la creación de espacios en los cuales los profesores del Departamento, junto a los estudiantes, participaron en el diseño, planificación y ejecución de los ciclos de cineforos.

Es importante destacar que el método de la investigación-acción es concebido como un proceso cíclico, con diferentes instancias en la formulación y resolución del problema a investigar. Generalmente, la investigación-acción comienza con un diagnóstico del problema, progresa hacia la planificación y diseño de estrategias de intervención, implementa la acción, para luego comparar con el diagnóstico inicial.

Coghaln y Brannick (2001), destacan que el componente de cambio social es la característica más resaltante de la metodología basada en la investigaciónacción. Para estos autores, este tipo de modalidad investigativa busca contribuir simultáneamente a generar conocimien- to y a lograr cambios en la vida social diaria del grupo al cual está dirigida la acción de cambio. En nuestro caso en particular, la acción más resaltante estuvo dirigida a emplear métodos de enseñanza alternos y complementarios a los tradicionales con el fin de lograr aprendizajes vivenciales y significativos tanto en los estudiantes como en los docentes y por otro lado, generar ingresos propios en beneficio del Departamento de Psicología.

Las autoras de la presente investigación hicieron un diagnóstico previo que involucró a los alumnos de la mención Orientación de la Escuela de Educación de la Facultad de Humanidades y Educación de la Universidad del Zulia y a los profesores del Departamento de Psicología (dependencia universitaria que administra la mencionada mención). Este diagnóstico previo condujo a la identificación de dos requerimientos: a) Necesidad de generar actividades que condujeran a la obtención ingresos propios, de modo tal que fuese el Departamento quien controlara y administrara el destino de estos posibles ingresos; b) Necesidad de crear situaciones didácticas que facilitasen aprendizajes sobre la complejidad de las relaciones humana y que fuesen diferentes a los logrados con metodologías tradicionales.

De estos dos requerimientos, surgió el concepto de cinemaeducación y la estrategia del cine foro como objeto de estudio a investigar. Partimos de la información que los mismos estudiantes y docentes nos facilitaron.

Desde esta perspectiva, la tarea de diseño de los ciclos de cineforos consistió en programar, organizar y secuenciar las acciones didácticas que propiciasen cono- 
cimientos nuevos y significativos, así como las de generación de ingresos propios.

También fue necesario que los nuevos conocimientos que queríamos facilitar con los ciclos de cineforos pudieran relacionarse de forma sustantiva y no arbitraria con lo que ya conocían los alumnos, debiendo adoptar una actitud activa para establecer dichas relaciones. Buscamos que en el proceso de participación en los cineforos se dieran estas tres condiciones: 1. Que los nuevos materiales para el aprendizaje fueran potencialmente significativos, es decir suficientemente sustantivos y no arbitrarios para ser relacionados con las ideas relevantes que poseyeran los alumnos sobre la orientación y la psicología y que se relacionaran con los contenidos inherentes a las asignaturas que administra el departamento. Así, seleccionamos dos temas, el amor y la familia, como ejes organizadores sobre los cuales giraron las películas y su posterior discusión 2. Que se generara una actitud activa en los alumnos en cuanto a su participación en la organización y desarrollo de los cineforos, teniendo en cuenta la importancia de los factores de participación, atención y motivación; 3. Que se generaran interacciones entre la nueva información suscitada en los cineforos y las ideas relevantes ya existentes en la estructura cognitiva de los estudiantes. En este sentido, nos aseguramos que los profesores utilizaran las películas y su posterior discusión como insumo en sus clases.

Nuestro punto de vista sobre el cineforo como estrategia didáctica subrayó la utilidad de integrar una cuádruple significatividad en los aprendizajes: 1) Para los es- tudiantes, 2) Para los profesores, 3) Para el propio diseño curricular, sobre todo en aquellas asignaturas que tienen entre sus contenidos el estudio de la pareja y la familia como sistema humano, en el marco de la formación de orientadores, y 4) Para el Departamento de Psicología, como actividad generadora de ingresos propios.

Una vez realizado el diagnóstico de la situación-problema, es decir la necesidad de implementar estrategias didácticas extramuros que al mismo tiempo fuesen capaces de generar ingresos propios, pasamos a la etapa de la planificación de actividades. En esta etapa decidimos la realización de los ciclos de cineforos, con el fin de investigar si la cinemaeducacion, implementada a través de ciclos de cineforos y como actividad didáctica exploratoria extramuros, era capaz de generar ingresos propios y servir de insumo para favorecer aprendizajes diferentes y complementarios a los logrados con tecnologías de enseñanza tradicionales.

Realizamos dos ciclos de cineforos, compuestos por cuatro películas por ciclo, para un total de dieciséis horas por cada ciclo. Una vez finalizado cada ciclo, evaluamos el desarrollo de la actividad, con el fin de mejorarla y compararla con los propósitos iniciales.

\section{Contexto de la planificación de los cineforos}

Los profesionales del campo educativo en general y de la orientación y la psicología en particular tienen la responsabilidad de revisar sus prácticas pedagógicas a la luz de las transformaciones que, a manera de reto, imponen los cambios sociales; así mismo, existe una ne- 
cesidad de conceptualizar estos momentos como situación contextual para los procesos de enseñanza y de aprendizaje, así como encontrar estrategias didácticas acordes a nuestras disciplinas, a partir de las cuales redefinir las intervenciones psicoeducativas.

Partimos de la consideración de que el gran desafío del siglo XXI es la adecuación de los sujetos a la transformación paradigmática, lo que implica ser productores de innovaciones y desarrollar las competencias necesarias para interactuar en un medio, en donde los cambios se aceleran vertiginosamente. Pensamos que la cinemaeducación, o el empleo del cineforo en la enseñanza de la orientación permite lograr esos cambios.

Ello nos situó, como docentes del Departamento de Psicología, ante el desafío de generar una propuesta educativa capaz de articular diferentes aspectos de dicha problemática de manera coherente.

Para introducirnos en la temática de la cinemaeducación, era necesario la realización de una experiencia concreta por nuestra parte, con la participación activa de los alumnos, que nos permitiera analizar el desempeño de los grupos en diferentes condiciones de tarea y modalidades de interacción, reconociendo las especificidades de las modalidades intramuros (clase tradicional) y extramuros (actividad de cineforo).

En cuanto a los aspectos académicos, los cineforos deberían cubrir el espacio curricular de las cátedras que administra el Departamento de Psicología, tanto en la Escuela de Educación en general, como en la mención Orientación en particular.
Para la organización de los cineforos, seguimos la guía esquematizada por Pinterits y Atkinson (1998), quienes recomiendan hacer una cuidadosa selección de las películas antes de decidir utilizarlas en la enseñanza de la orientación. La selección de las películas la hicimos tomando en cuenta las necesidades temáticas de las asignaturas que dictan los profesores del Departamento de Psicología. Una vez seleccionadas las películas, los profesores del Departamento las vimos en privado y discutimos las tramas y personajes de cada una de ellas, así como también visualizamos la información que cada film nos aportaba para luego discutirlo en nuestras respectivas cátedras.

Nuestra intención como responsables de la organización de los ciclos de cineforos estuvo centrada en que los docentes incorporaran a sus cátedras los aspectos discutidos en los cineforos. También decidimos abordar la complejidad del hecho educativo y su multideterminación, donde lo macro atraviesa lo micro.

Además de estudiar la incidencia de la cinemaeducación en el aprendizaje de temas relacionados con orientación; con la implementación de los ciclos de cineforos buscamos generar ingresos propios que beneficiasen al Departamento de Psicología.

\section{Proceso de aplicación}

\section{Ciclos de cineforos}

Cada ciclo incluyó la proyección de cuatro películas por ciclo, a razón de una película semanal. Una vez proyectada cada película fue analizada y discutida. 
El Primer Ciclo de Cineforo versó sobre la temática de "El Amor y la Relación de Pareja”, y las cuatro películas seleccionadas fueron: Ojos Abiertamente Cerrados (Eyes Wide Shut), Amores Perros, El Marqués de Sade y Lucía y el Sexo. El Segundo Ciclo de Cineforo se centró en la "Familia", y las películas proyectadas fueron: Claroscuro, El hijo de la Novia, Belleza Americana (American Beauty) y Como Agua para Chocolate.

Una vez finalizada la proyección de la película en cada una de las sesiones, un panel de expertos comentó sobre los procesos socio-emocionales que se pudieron apreciar en las relaciones presentadas en el film. Posteriormente, se invitó a los asistentes a realizar preguntas y exponer inquietudes y comentarios sobre la película y las ideas suscitadas con ella.

\section{Población}

Los Ciclos de Cineforos estuvieron dirigidos al público en general, pero muy especialmente a la comunidad estudiantil de LUZ. Se dio preferencia en primer término a los estudiantes de la mención Orientación de la Escuela de Educación de la Facultad de Humanidades y Educación y en segundo orden a los de las otras menciones de las Escuelas de Educación, Trabajo Social, Medicina y Sociología.

El número de participantes asistente fue de aproximadamente doscientas personas por cada ciclo completo. Es importante acotar que la inscripción se realizó antes de comenzar el ciclo e incluyó las cuatro películas que lo conforman; es decir no se permitieron inscripciones para una sola película.

Facilitadores

Cada sesión del cineforo contó con un panel de al menos tres expertos, pro- fesionales universitarios invitados de otras instituciones y de diferentes áreas del conocimiento, quienes analizaron desde sus respectivos campos del saber cada una de las películas. Nos aseguramos de que en el panel que discutiera la película hubiese al menos un profesor del Departamento de Psicología.

De igual modo, la mayoría de los profesores del Departamento de Psicología participaron no solo como organizadores y panelistas, sino también como participantes activos en la discusión que se suscitó después de proyectada cada película.

Por otro lado, también participaron los cursantes de la Maestría en Orientación de la División de Estudios para Graduados de la Facultad de Humanidades y Educación de la Universidad del Zulia, con el fin de integrar el post-grado con el pre-grado. En este sentido, se incluyeron en el panel, alumnos destacados de la referida Maestría.

\section{Análisis de la experiencia}

En este apartado dividimos el análisis de los aportes que han ido conformando los pilares del cineforo en dos categorías: a) como estrategia didáctica y b) como actividad generadora de ingresos propios. Es importante resaltar que los ciclos de cineforos se propusieron como una actividad exploratoria y por lo tanto los resultados definitivos necesitarán ser evaluados en el tiempo.

\section{a) El cineforo como estrategia di- dáctica}

Los docentes se mostraron abiertos a la experiencia de emplear las discusiones generadas en el cineforo como in- 
sumo en la enseñanza de contenidos relacionados con sus materias.

Los profesores reportaron que sus alumnos tuvieron discusiones en sus respectivas asignaturas sobre lo expuesto por los panelistas en cada película, al tiempo que los alumnos reportaron que no solo se nutrieron de las exposiciones de los panelistas, sino que también se generaron aprendizajes significativos de los comentarios y preguntas hechos por sus pares, tanto en las discusiones realizadas después de proyectada cada película, como en las que se llevaron a cabo en el salón de clase.

Por ejemplo, los profesores de las asignaturas relacionadas con el manejo y estudio de casos en orientación, tales como "Orientación Personal", "Sistemas Humanos", "Orientación Familiar" y "Pasantía", y con las materias denominadas de "servicio", tales como "Psicología del Desarrollo y "Psicología Educativa", emplearon al cineforo en las discusiones de casos de sus respectivas materias. Tanto los docentes como los alumnos reportaron que ellos enfocaron las películas como "casos de la vida real que nos podrían llegar para que los atendiésemos como orientadores".

Las discusiones que realizaron los profesores estuvieron basadas en la propuesta presentada por Sarah y Carl (2000). Estos autores proponen la utilización del cineforo en la educación de orientadores en cuatro rubros : a) El uso del cine para enseñar diagnósticos; b) La enseñanza de las teorías y estrategias de la orientación a través del cine; c) Cine y ética; y d) Cine y procesos de orientación.

Por ejemplo, en la cátedra "Orientación Personal", el docente utilizó la pelícu- la "Claroscuro", como si fuese un caso real que los estudiantes debían asumir como orientadores. Él discutió con sus estudiantes sobre los enfoques más apropiados para trabajar en el caso (personaje protagonista del film). También discutieron las ventajas y desventajas de diferentes enfoques de orientación que los panelistas expusieron sobre el caso y el grupo llegó a la conclusión de que el psicoanálisis clásico no era el enfoque de intervención más indicado, y sí una terapia de tipo narrativa. Así mismo, el docente utilizó esta película como insumo en la evaluación de sus alumnos. Les pidió que escribieran una monografía de aproximadamente 6 páginas, donde enfocasen las reacciones personales y éticas de cada estudiante, situándose éste como orientador del personaje principal de la película.

De igual modo, en la asignatura "Orientación Familiar", la profesora utilizó las tramas de algunas de las películas proyectadas para ejemplificar los conceptos de pensamiento lineal, típico de la psicología individual y pensamiento sistémico, esencial para abordar la estructura de una familia.

Por otro lado, como actividad intramuros, la docente de esta misma asignatura solicitó a sus alumnos que analizaran las discusiones de cada película. El Cuadro 1 presenta un resumen de los productos generados por los alumnos de la asignatura Orientación en Familia, en relación a los contenidos de las películas. El Cuadro 1 podría considerarse como una incipiente base de datos que sistematiza películas, contenidos y posibles discusiones sobre temas relacionados con esta asignatura. 
Otro de los logros importantes generados con las discusiones del cine foro se relacionó con las distintas exposiciones que hicieron los panelistas sobre cada película. Por ejemplo, los alumnos de la asignatura “Orientación Familiar' reportaron que fue muy enriquecedor el poder participar en la discusión con diferentes expertos de otros campos distintos al de la orientación. Así, en la película Belleza Americana, el panel de expertos no solo analizó los personajes desde el punto de vista psicológico y familiar, sino que se utilizó la trama para mostrar los aspectos "decadentes", y "vacuos de sentido" de un sector de la clase media norteamericana, de tal modo que de la discusión de la película, los alumnos de la materia "Orientación Familiar" acuñaron la enseñanza de que el dinero, el confort y el consumismo no hacen felices a los seres humanos, al tiempo que pudieron valorar la importancia de la familia en el ciclo de vida de los seres humanos.

Los docentes también resaltaron el hecho de que los ciclos de cineforos les permitieron a sus alumnos adquirir competencias en el área de orientación personal y familiar en un ambiente mucho más relajado y seguro, puesto que el cine genera vivencias muy parecidas a las que se producen en un caso real, pero sin la presión que sienten los estudiantes de "tener que hacer algo para ayudar al caso". A pesar de lo "ficticio" del cine, tanto docentes como alumnos significaron que las películas seleccionadas las abordaron como "casos reales", pero sin "riesgos reales". Esta técnica ha sido descrita por otros investigadores de la cinemaeducación como desplazamiento (Higgins y Dermer, 2001), puesto que le permite al estudiante involucrarse emocionalmente con los personajes y tramas de la historia (es decir "vivir y "sentir" la película), pero sin consecuencias iatrogénicas para los personajes. De este modo, las películas proyectadas sirvieron de insumos "reales" para que los alumnos discutieran casos "casireales".

Igualmente, los ciclos de cine foros sirvieron para ejemplificar conceptos complejos y difíciles de enseñar, como por ejemplo el de la intersubjetividad en la orientación. Los cineforos fueron empleados por los profesores para analizar las reacciones de los estudiantes ante las películas, y compararlas con casos reales en orientación.

Así como un caso en orientación puede evocar intensos sentimientos en el orientador, las películas también suscitaron fuertes sentimientos en los alumnos, y los ciclos de cineforos fueron utilizados para ejemplificar esas reacciones personales que los orientadores tienen frente a sus casos, resaltando el valor que esta información puede tener como estrategia epistemológica en la orientación.

\section{b) El cineforo como actividad ge-}

\section{neradora de ingresos propios}

Dentro de los beneficios más resaltantes que se han obtenido del cineforo como actividad generadora de ingresos propios, basada en la participación, se pueden mencionar que la más resaltante fue la participación masiva de estudiantes de la mención Orientación y de otras menciones de la Escuela de Educación.

Como actividad generadora de ingresos propios, el primer ciclo de cineforo reportó una inscripción de 227 participan- 


\section{Cuadro 1}

\section{Películas y temas relacionados con la asignatura "Orientación Familiar"}

\begin{tabular}{lll} 
Película & Temas Sobresalientes Trama & $\begin{array}{l}\text { Enseñanzas/reflexiones } \\
\text { más importantes }\end{array}$ \\
\hline
\end{tabular}

Ojos abiertamente cerrados

Fantasías en la relación de pareja, salud sexual de la pareja, infidelidad. Perversiones de la vida postmoderna en cuanto a la sexualidad

\section{Amores perros}

\section{El Marqués} de Sade

Lucía y el sexo El amor, los duelos, la fidelidad, la importancia de la narración en el amor y la relación de pareja. El sexo. Nuevas formas de enfrentar el hecho cinematográfico
Una pareja norteamericana de clase media alta se enrumba, cada uno por su lado, en narrar sus fantasías eróticas con personas diferentes a la propia pareja

Tres historias de familias de diferentes clases de la sociedad mexicana despliegan sus sufrimientos y añoranzas por el amor.

Un hombre del siglo 17 narra sus fantasías eróticas de forma tal que es expulsado de la sociedad y puesto en prisión.

Una mujer común y corriente, se enrumba a convivir con un hombre extraño del que se enamora profundamente; por sus propios conflictos relacionales, éste no puede responder a las necesidades de su pareja
La comunicación en pareja es importante, pero tiene límites. La fantasía no es igual a la realidad. La pareja sobrevive a la infidelidad (sea esta en la fantasía o en la realidad)

Los seres humanos somos vulnerables al amor, sea cual sea nuestra clase social

La sociedad y época histórica en que vivimos moldea lo permitido en el amor

Si no conocemos nuestra historia, estamos condenados a repetirla 


\section{Cuadro 1. (Continuación)}

\begin{tabular}{lll} 
Película & Temas Sobresalientes Trama & $\begin{array}{l}\text { Enseñanzas/reflexiones } \\
\text { más importantes }\end{array}$ \\
\hline
\end{tabular}

\section{Claros-curo}

\section{El hijo de la} novia

\section{American \\ Beauty \\ (Belleza \\ America-na)}

\section{Como agua para chocoloate}

Sobreprotección, psicosis, violencia familiar, genio y locura

Mal de Alzaheimer, incapacidad en mantener relaciones de pareja, valores familiares, cardiopatías, tabaquismo, adicción al trabajo, reconciliación con uno mismo

Crítica a la superficialidad de la familia de clase media americana, pedofilía, homosexualidad y normalidad, muerte, superficialidad e insatisfacción en la vida cotidiana moderna, aceptación de los miembros de la familia

Tradiciones familiares. Una familia mexicana Mujeres y poder. Papel de la feminidad en la sociedad mexicana de principios del siglo 20
Un músico de inteligencia excepcional sucumbe a la psicosis. Su padre es un hombre que mentalmente lo asfixia, al punto que el protagonista enloquece y pasa 10 años de su vida en una institución para enfermos mentales

Una familia de clase media argentina enfrenta varios dilemas de la vida moderna: la enfermedad de Alzaheimer, la cardiopatía del hijo de cuarenta años, la venta del negocio familiar que los definió como familia, el destino de la familia

Una familia norteamericana de clase media narra sus insatisfacciones y carencia de sentido de la vida moderna

somete a su última hija a la tradición familiar: no se puede casar pues debe cuidad a su madre hasta que ésta muera y debe renunciar a cualquier expresión de vida afectiva en pareja
El amor y la superación nos ayudan a vencer hasta los males mas miserables de nuestras vidas. Para entender la locura hay que acercarse a lo más esencial de la vida.

La única manera de conseguir la felicidad, es reconciliándose con uno mismo y enfrentando los fantasmas del pasado en el aquí y el ahora

Si queremos ser felices, tenemos que encontrar la esencia en los detalles comunes y diarios de nuestra vida. La superficialidad nos lleva a la insatisfacción y a la entropía social.

La mujer necesita encontrarse a si misma, a través de la historia y de su propio descubrimiento.

Fuente: Elaboración propia. 
tes quienes cancelaron Bs. $6.000,00$ cada uno por el ciclo completo de cuatro películas; lo que produjo un ingreso de Bs. $1.362 .000,00$ de los cuales quedó una ganancia de Bs. 724.000,00; una vez cubiertos todos los gastos generados por la actividad. La ganancia generada permitió la compra de un equipo de DVD para las proyecciones de los siguientes ciclos y actividades académicas, un televisor, un equipo de sonido y material de oficina para el departamento.

El segundo ciclo de foro contó con la inscripción de 233 participantes a Bs. $8.000,00$ cada uno, por el ciclo completo de cuatro películas. En esta oportunidad el ingreso fue superior al anterior, Bs. 1.864.000,00; sin embargo los gastos se incrementaron por el aumento en los precios de los insumos utilizados y en el alquiler del auditorio donde se realizó el ciclo. La ganancia de toda la actividad fue de Bs. $835.000,00$ la cual fue invertida en una impresora multiuso para el Departamento, con función fotocopiadora, escaner y fax; otro equipo de sonido portátil para las actividades académicas de la mención orientación y las clases de psicología y materiales de oficina tales como carpetas, archivadores, resmas de papel bond, tinta para impresoras.

Los beneficios económicos generados a través de la actividad del cineforo redundaron no sólo en la adquisición de equipos y materiales de oficina, sino también en el involucramiento, participación y compromiso del personal docente que labora en el Departamento y de los estudiantes de los últimos semestres de la mención, quienes colaboraron en la logística y organización de las actividades.
Los profesores reportaron haber consolidado el compromiso y la responsabilidad de generar actividades de promoción y generación de recursos económicos para el Departamento, que puedan ser invertidos en mejorar las condiciones en las que se desarrolla el proceso enseñanza-aprendizaje, a través de actividades innovadoras como éstas.

Durante la evaluación de las actividades que conformaron los ciclos de cineforos, los docentes manifestaron haber afianzado el trabajo en equipo tanto en el diseño, como en el desarrollo y evaluación de las actividades. Este logro se corresponde con el concepto de participación planteado por Velasco (2002) que sirvió de fundamento para la elaboración de la propuesta del cineforo como estrategia para generar ingresos propios.

Por otro lado, tanto docentes como alumnos coincidieron en que esta actividad produjo beneficios mutuos tanto a nivel económico para el Departamento de Psicología como para los propios estudiantes quienes con el monto significativo de la inscripción analizaron una serie de elementos relacionados con los contenidos de al menos una de las asignaturas que están cursando, desde diferentes perspectivas y puntos de vista, pudiendo aclarar dudas, manifestar sus inquietudes y expresar sus propias reflexiones y conclusiones.

Las discusiones realizadas posteriormente en la asignatura Psicología del Desarrollo por uno de los profesores, reporta que el involucramiento de los estudiantes y docentes a esta actividad ayudó a desarrollar la capacidad de análisis y síntesis de los procesos estudiados en 
las películas y a proponer otro tipo de actividad generadora de ingresos propios, antes no contempladas. Así mismo, los ciclos de cineforos sirvieron de ejemplo para continuar realizando actividades para generar ingresos propios: el grupo de estudiantes de la asignatura Práctica III en Orientación, bajo la coordinación de su profesora asesora, ofrecieron posteriormente un ciclo de talleres vocacionales a los estudiantes de noveno grado de dos instituciones educativas, generando ingresos para el Departamento.

De tal manera que con este tipo de actividad, se contribuyó con el proceso de generación de ingresos propios que busca la Nueva Gestión Pública (NGP); porque el Departamento produjo y administró sus propios ingresos en pro del mejoramiento de la calidad de la educación.

Es preciso acotar que es la primera evaluación que se le hace a este tipo de actividad y es necesario continuar haciéndolo para contrastar los resultados que se van obteniendo, de manera que el Departamento siga avanzando hacia el logro de los objetivos previstos en materia de investigación en ciclos de cine foros en cuanto a propuestas de generación de ingresos propios y de ensayo de estrategias tecnológicas de enseñanza innovadora.

\section{Conclusiones}

Con la utilización de ciclos de cineforos se pretendió acercar varios aspectos relacionados con la enseñanza de la complejidad en las relaciones de pareja y el medio familiar, utilizando el cine como recurso a partir de la comunicación, la experiencia y el trabajo didáctico con la puesta en marcha de ciclos de películas en torno a temas comunes (la pareja y la familia) y facilitando al profesorado un material didáctico, práctico, útil y de sencilla aplicación para trabajar la enseñanza de la orientación y la psicología.

Los resultados iniciales de esta experiencia exploradora indican que la modalidad de cineforo es fructífera como actividad generadora de ingresos propios, con potencial para convertirse en un programa autogestionador de recursos financieros y como estrategia didáctica innovadora, con posibilidades de evolucionar hacia un programa de extensión universitaria, capaz de producir conocimientos sobre la complejidad de las relaciones psicológicas del ser humano, empleando estímulos visuales, auditivos y experienciales.

\section{Referencias Bibliográficas}

Alexander, Mat (1995), Cinemaeducation: An innovative approach to teaching multicultural diversity in medicine. Annual of Behavioral Science and Medical Education. No. 2.

Alfredo, Rosa (2004), Cine, Historia y Medicina. Suplemento No 1 de CONECTA, ISSN: 1576-4826. En www.dsp.umh. es/conecta/cmh/Cine.pdf - 112k. Consultado el 15-06-2004.

Barzelay, Michael (2004), La Nueva Gestión Pública. Un acercamiento a la investigación y al debate de las políticas. 1era. Edición. México. Fondo de Cultura Económica.

Coghaln, David. y Brannick, Teresa (2001), Doing Action Research in Your Own Organization. London, UK, Sage.

Davis, Keith y Newstrom, John (1994), Comportamiento Humano en el trabajo. México, Mexico, Mc Graw Hill. 
Flores, Ochoa (1994), Hacia Una Pedagogía Del Conocimiento. Bogotá, Colombia McGraw Gill.

Greenberg, Hir (1993), Screen memories. Hollywood cinema on the psychoanalytic couch. New York, USA, Columbia University Press.

Higgins, Jeannette y Dermer, Shannon (2001), The use of film in marriage and family education. Counselor Education and Supervision. American Counseling Association. Issue 3, March.

Kemmimis, Stephen y Taggart Robin (2000), Participatory Action Reserch. En Denzil, Norman and Liuncoln, Yvonna. (Eds) Handbook of Qualitative Research. Thousand Oaks, USA, Sage Publications.

Koch, Gary y Dollarhide, Colette (2000), "The use of cinema in the counselor education curriculum. Strategies and outcomes". Couselor Education and Supervision. American Counseling Association. Vol. 39, Issue 3, March 2000.

Nelson, ML.y Neufeldt, Sa. (1998), "The Pegagogy of Counseling”. Counselor and Education and Supervition. American Counseling Association. Vol 38, issues 2, p 70 .
Ochoa, Haydeé (2004), "Editorial en la mira. La nueva gestión pública". Revista Venezolana de Gerencia. Año 9, No. 25

Petrella, Ricardo (1997), El Bien Común. Elogio de la Solidaridad. Madrid, España, Ed. Temas de Debate.

Pintents, Janie y Atkinson, Donald (1998), "The diversity video forum: An adjunt to diversity sensitivity training in the classroom. Counselor Education and Supervision. American Counseling Association Vol 38, No. 3

Sarah, Mat y Carl Rank (2000), The use of cinema in the counselor education curriculo: strategies and outcomes. Counselor Education and Supervision. American Counseling Association Vol 40, Issue 2.

Velasco, Manuel (2002), Editorial. Revista de la Facultad de Medicina de la UCV. RFM Caracas Vol.25. No.2.

Vera, George (2002) (a), "Pedagogía y Formación de Orientadores: Una Perspectiva Constructivista". Revista de Pegagogía, No. 26, 2002.

Vera, George (2002) (b), "Perspectives on the Teaching of Core Counseling Courses: A Grounded Theory Inquiry". Tesis de Doctorado. Universidad de New Orleans. Sin publicar. 